can entertain a doubt as to its being the most absurd and dis. honest form of quackery and imposture ever foisted upon the public. But the fact, to which we would direct attention, is, the assumption of the Royal Court of Guernsey to grant licences to practise medicine and surgery. Were such licences limited to duly qualified practitioners, no blame might attach to this stretch of power, as the public would thereby be protected from deception and fraud; but the incompetency of the Court to judge of the authenticity and validity of assumed diplomas is proved by the fact, that unqualified individuals, and uneducated ai.venturers, on presentation of alleged diplomas from the "British College of Heal:h," \&c., or certificates, as "Officicrs de Santé," have obtained leave to practise. And Mr. OzıNve, in 18t0, on an alleged diploma, as "docteur en médecine" of the faculty of Paris, only, procured from the said court a licence to practise surgery. Nevertheless, with additional inconsistency, a licence to practise in Guernsey was refused a few months since to a graduate of Cambridge.

It is high time that the Apothecaries' Act should be registered in Guerrsey, as a protection to those who have voluntarily pursued the course of study prescribed-to those who have undergone the trouble and expense of examinations before the Medical and Surgical Colleges, and Society of Apothecaries, and who, from their education and respect. ability, are entitled to be protected from imposture and quackery.

\section{A Anirror}

OF THE PRACTICE OF

\section{MEDICINE AND SURGERY IN THE \\ HOSPITALS OF LONDON.}

\section{GuY's Hospital. \\ Amputation at the Shoulder-joint.}

(Under the care of Mr. BransBY Cooper.)

WE recently witnessed this important operation performed, by Mr. Bransby Cooper, on a woman, twenty-five years of age, who had been suffering for years from necrosis of the humerus. The history of the case, as noted down by Mr. Stocker, the dresser of the patient, is as follows:-Harriet $\mathbf{S}-$, a servant, of dark complexion, was admitted into Dorcas ward, in May, 1848, under the care of Mr. Aston Key. She stated, that for no less than twelve years she had been suffering from necrosis of the humerus, and that the disease had originally been caused by a fracture of that bone. She had been a patient in Guy's Hospital eight years previous to her admission in 1848, when Mr. Key had operated upon her, and had removed two pieces of dead bone. During this interval she had otherwise enjoyed good health, and the arm being now carefully examined, Mr. Key determined to remove the necrosed portions of bone. The patient, having been placed under the influence of chloroform, a crucial incision was made over the upper part of the external condyloid ridge of the humerus, and the integuments dissected back. The bone being thus laid quite bare, several sequestra were removed with the chisel and hammer.

One month after this operation, the wound was quite healed, som : more pieces of bone having come away during the suppurative stage. She remained in the hospital for two months after cicatrization, and $\mathbf{M r}$. Key, then finding that the upper part of the humerus was also diseased, operated in that region likewise, but removed only a very small portion of necrosed bone. She soon recovered from this second operation, and went out nearly well.

The patient had, however, as is but too often the case, ex. perienced only temporary relief, and she applied again for admission in December, 1849 , when she was placed under the care of Mr. Bransby Cooper. She now complained of great pain in the arm, increasing so much at night as to destroy her rest completely. The limb presented a number of apertures of simuses, with the usual inverted edges and a light-yellow discharge, and the probe introduced into one of them in the upper part of the arm readily detocted a moveable piece of necrosed bone. One month after this last admission, Mr. Cooper removed a sequestrum, about three inches in length, from the superior portion of the humerns, and the patient expressed herself considerably relieved by this measure; the pain diminished notably, and she remained in a tolerable condition for about three weeks, when the arm and shoulder became again excessively painful, so much so as to entirely prevent her from sleeping. $\mathrm{Mr}$. Cooper perceiving that the girl's health was being undermined by this protracted affection, and fearful that the disease might extend to the joint, proposed amputation at the scapulo-humeral articulation, and the patient very readily submitted to the necessity of the case.

She was brought into the theatre on the 12 th of March, and refused to inhale the chloroform, stating that she knew she must undergo the operation, and would have the fortitude to go through it. Though such courage calls for great admiration, it is alnost a pity that pain should be endured when we possess such a ready means of avoiding it. The ratient being seated, Mr. Cooper made his first incision, with a large-sized knife, in a longitudinal direction, commencing a little externally to the acromion, and descending in a curved manner to about four inches from that process near the in. sertion of the deltoid. The second incision was made with the left hand, and began a little internally to the acromion, run downwards, and terminated at the extremity of the first cut. The deltoid being thus comprised by these two incisions, was rapidly dissected upwards towards the head of the humerus, and the flap being held by an assistant, the tendon of the biceps, those attached to the head of the bone, and the capsule, were diviled with a similar celerity. The humerus having been disarticulated, Mr. Cooper inquired whether the assistants were ready, and with one sweep of the knife divided the soft parts situated interually. From the beginning of the operation, Mr. Birkett exercised steady compression on the subclavian artery with a padded key, and the flap containing the axillary artery being seized immediately the muscles were divided, the circulation was thus so completely commanded that the hæmorrhage was very trifling. The axillary artery was immediately secured, with a few other vessels of lesser importance, the flap brought into contact with the parts next to the trunk, the wound lightly dressed, and the patient carefully removed to her bed. From the remarkable celerity with which this amputation was performed, we should be inclined constantly to give the preference to this mode of operating. There seems to be more certainty in making a square or circular flap at the cushion of the shoulder than to thrust the double edged knife through the base of the deltoid, and run it downwards to the insertion of the muscle. The nature of the injury for which the operation is performed will very often leave the surgeon no choice, and oblige him to take a flap where he can; but when there is a choice, the advantage seems to lie on the method which Mr. Cooper adopted. The patient has progressed very favourably up to this day; most of the ligatures have come away, the wound has healed by first intention, and the stump is of a very regular form. On examining the limb, it was fonnd, that from the condyles up to the centre of the shaft, the bone was pretty healthy; the rest of the shaft was perforated by three large holes, in one of which lay a loose sequestrum. The head of the humerus was severely attacked, the cartilage partly gone, and the neck almost, destroyed. The two upper perforations communicated with each other, the medullary canal being thereby much encroached upon. The glenoid cavity was, however, free from disease.

\section{Royal Free Hospitat.}

Endocarditis and Cirrhosis of the Liver.

('inder the care of Dr. Hedtr.)

Dr. Heale had lately in his wards a patient whose case afforded various features of interest. The patient was only twenty-three years of age, and presented a series of affections 
which, though in some degree depending on one another, offered a rather unusual assemblage at that early period of life. When admitted, the patient had had a cough only for two months, and presented a suffused, dark, and swollen face; he breathed very short, and the abdomen was much distended and tympanitic, particularly above the umbilicus, where the flatus seemed very near the surface. Fluctuation was made out by percussing across the abdomen, from between the ilium and ribs on one side, and the same spot on the other: the fluid was therefore situated behind the intestines. On auscultating the heart, a lond, coarse bruit was heard with the first sound, and percived with the greatest intensity four inches below the nipple. The heart, being evidently hypertrophied, occupied a large space, and a great and visible impulse accompanied each stroke of the organ; the hand, placed on the cardiac region, experienced a purring sensation, and the same feeling was conveyed to the ear by the stethoscope placed be neath the left scapula. In the lungs, pnerile respiration was observed on both sides anteriorly, and the posterior portions gave large and small crepitation, and moist rhonchi; pulse, 100 ; tongue moist; urine, specific gravity 1.025 , with a deposit of lithates, though quite clear on boiling; expectoration brownish. Dr. Heale diagnosed the case as one of endocarditis, attended with ascites and pneumonia, but the exact cause of the great degree of tympanitis could not be made out, and was revealed only by the autopsy. Dr. Heale, after having obtained free evacuntions from the bowels, prescribed small doses of mercury, the most approved diuretics, and had blood drawn by cupping from the cardiac region. When the gums were tender, about six days after admission, the symptoms were somewhat mitigated, and the patient was put upon ammonia, and small doses of mercury with chalk. About ten days after the original examination the first sound of the heart was observed to be ill-defined, and, as it were, split into two sounds; and the second exaggerated into a noise resembling a loud knock, but no whizzing of regurgitation was heard. The tympanitic distention beneath the sternum was now so prominent, and the skin so dense, that it almost seemed as if the Hatus were external to the abdominal muscles. Twelve days after admission general anasarca came on, the fluctuation in the abdomen became rather more distinct, and Mr. Thomas Wakley was consulted on the propriety of paracentesis. Mr. Wakley was of opinion that, cedema of the ab. dominal parietes being present, the operation did not promise that relief to the patient which would warrant its adoption. The poor fellow died exactly one month after his reception into the house.

Autopsy. - Abdomen very prominent and tympanitic, general anasarca. On an incision being made, a large quantity of flud escaped from the abdominal cavity: it issued from above the stomach, and behind and below the intestines, which latter were occupying the anterior part of the abdomen. The stomach was greatly distended, and filled the epigastric region, and the omentum considerably thickened, and, covering the intestines, was firmly bound down to the pubes by its lower margin. The liver was found very much enlarged, granular, and hard, exhibiting, in a striking form, an early stage of cirrhosis; the pancreas, which was hard, knotty, and rigid, appeared to partake of the same morbid character as the liver. The kidneys were healthy; the lungs were much congested, and spotted over the anterior surface, as in purpura; on examining the spots carefully they were found to have been produced by pulmonary apoplexy; in the posterior portions, the latter phenomenon was much more distinct, clots of the size of walnuts being observed. In fact, the whole of the third lobe of the right lung consisted of one solidified mass, of a dark blue colour, not crepitating on pres. sure, and sinking immediately in water. The heart occupied about double the ordinary space; its walls were, however, not thicker than those of the healthy organ, and were therefore exceedingly weak, in proportion to the size of the viscus. The pulmonary semilunar valves were healthy, but those of the aorta extremely thickened, and deficient in flexibility, yet not rough on their surface. Both the tricuspid and mitral valves were of the consistence of cartilage, scarcely flexible, and al. most ossified.

The points of interest in this case seem, first, the tympanitis which resulted from the adhesion of the lower edge of the omentum to the inferior boundary of the abdomen. This abnormal connexion, so entirely confining the stomach, prevented its ever once contracting, during the illness, for the purpose of expelling the flatus which it contained. It likewise prevented the bowels from floating upwards. The fluid could therefore not have been evacuated, by tapping without wounding both the omentum and intestines. The condition of the liver and pancreas must have been mainly instrumental in the production of ascices, the general anasarca supervening when the heart had reached the maximum of hypertrophy. The pulmonary apoplexy, the result of the cardiac disease, being exhibited so strikingly, in three different forms-viz., spots, clots, and consolidation-was a feature worthy of particular attention. Most persons would have been disposed to deny that the diffused spots on the surface, and the total solidification of the whole middle lobe, proceeded from pul monary apoplexy, were not that fact confirmed by the evident disease, in its ordinary form, being present in another part of the lung. It should be carefully noticed, likewise, if we turn now to the heart, that as the auriculo-ventricular valves became thickened, and conscquently smooth, they ceased to give the rough sound of regurgitation, though the latter, of comrse, continued the same as before. Attention should likewise be given to the peculiar double sound into which the first sound of the heart seemed split, which in all probability proceeded from the ventricles not contracting synchronously during the systole, which circumstance was perhaps owing to the resistance offered by the valves to the direct current of the blood, though no sound of regurgitation was then produced.

Although the patient spoke of having suffered but two months previous to admission, it is evident that organic changes must have gone on for a considerable time with him. This would tend to show how prudent it would be, whenever we are consulted for apparent trifles, to inake a thorough examination of the state of the principal organs.

\section{King's College Hospttal. \\ Rhinoplastic Operation.}

(Under the care of Mr. Fergussox.)

This operation may be looked upon as the first step of plastic surgery, which latter has made such progress since the time of Taliacotius; and there is no doubt that the deformity which it is intended to correct is one of a most disagreeable nature. The value of rhinoplasty will be much enhanced, if it is considered that, besides making a new nose, it may have some influence in improving the voice, and perhaps also the smell.

Mr. Fergusson performed this operation, on the 16 th of February, on a man of about forty, who had been under his care several times before. His nose had suffered very much, and nothing but a shrivelled, flattened, and compressed little mass, was seen in the site of the original organ, the left ala being most depressed. This destruction had orjginally been caused by a blow, from which the ossa nasi and cartilages had suffered severe injury. The parts had healed very slowly, and Mr. Fergusson, a twelvemonth ago, had made incisions near the eyebrows for the removal of necrosed bones. At that time the man was pronised that a new nose would be made for him. He had gone into the country, where he recruited his health; and now had come to have the operation performed.

Mr. Fergusson gave, in this instance, the preference to the Indian method; and the patient having been placed under the influence of chloroform, a triaugular piece of leather, cut into the shape of the new organ, and made to suit the irregularities of the stump, was spread flat upon the forehead, with its base uppermost; deep incisions, with subsequent parings, were then made along the margins of the deformed nose, following the line where the sides of the flap, to be presently cut from the forehead, were to be implanted. When the paring had been carefully and regularly done on both sides, Mr. Fergusson cut out the skin and cellular tissue of the forehead, down to the periosteum, according to the shape above mentioned, and this being carefully dissected from above, downwards, to the root of the nose, where the dissection was carried decp, to render the vascular connexion more extensive; the flap was twisted on itself, and its edges were lrought into contact with the grooves previously made. An opening was here seen into the frontal sinus from the old exfoliation. The hæmorrhage was rather considerable, and somewhat increased the already great amount of trouble which this operation entails upon the surgeon; the sutures were, however, very neatly applied, three on each side; they kept the transplanted structure very steadily in situ, and the angles of the raw surface on the forehead were likewise approximated by sutures. The cavity of the new nose, which was partly supported by the shrivelled remaing of the old, was borne up by a small quantity of lint; the same was likewise put on the raw surface of the forehead; the 